\title{
Invariant measure in hot gauge theories
}

\author{
O.A. Borisenko, ₹ \\ Institut für Kernphysik, Technische Universität Wien, \\ Wiedner Hauptstr. 8-10, A-1040 Vienna, Austria \\ J. Boháčik, 円ُ
Institute of Physics Slovak Academy of Sciences, 84228 Bratislava,
Slovakia
}

\begin{abstract}
We investigate properties of the invariant measure for the $A_{0}$ gauge field in finite temperature gauge theories both on the lattice and in the continuum theory. We have found the cancellation of the naive measure in both cases. The result is quite general and holds at any finite temperature. We demonstrate, however, that there is no cancellation at any temperature for the invariant measure contribution understood as $Z(N)$ symmetrical distribution of gauge field configurations. The spontaneous breakdown of $Z(N)$ global symmetry is entirely due to the potential energy term of the gluonic interaction in the effective potential. The effects of this measure on the effective action, mechanism of confinement and $A_{0}$ condensation are discussed.
\end{abstract}

\footnotetext{
${ }^{1}$ Work was partially supported by Bundesministerium für Wissenschaft, Forschung und Kunst of Austria and by International Science Foundation Grant K4W 100.

${ }^{2}$ email: oleg@is1.kph.tuwien.ac.at

${ }^{3}$ email: bohacik@savba.savba.sk
} 


\section{Introduction}

During the last fifteen years a permanent attention was paid to the role of the invariant measure (IM) for $A_{0}$ gauge field in hot gauge theories [1, 2, 3, 4, 5, 6, 7, 8, 9]. Up to now, there is no a common opinion on effects of the IM with respect to such phenomena as confinement, $A_{0}$ condensation, etc. The first calculations by N. Weiss [1] showed that the 1-loop contribution of the longitudinal gluons cancelled the tree level of the IM decomposition around the constant classical value of the $\left\langle A_{0}\right\rangle$ field. A similar cancellation beyond the leading order was proven in [2]. Other arguments supporting this result can be found in [3], where it was suggested that the cancellation could be seen in dimensional regularization to an arbitrary order, so the same result should be valid in any scheme of the calculation. We gave already some comments on this topics in the review [5], where we argued in favour of this cancellation, though our arguments were rather speculative. However, conclusions deduced from this fact are different in [5] and in [1, 3].

Quite a different opinion has been advocated in [4, 6, 7] where it has been argued that there is no cancellation of the IM beyond 1-loop order. Also, the effective potential (EP) for the Polyakov loop calculated in $S U(2)$ lattice gluodynamics [8] shows that the vanishing value of the Polyakov loop in confinement phase is due to the IM term in the potential.

Let us now consider a possible physical interpretation of the IM contribution. Usually, two phenomena are pointed out which could be affected by the measure: the confinement of static quarks and the $A_{0}$ condensation. The confinement model based on the contribution of the IM was proposed in [4. Since it has been known that a flat integration measure fails to respect the $Z(N)$ global symmetry of the lattice action, it has been assumed to simulate the contribution of the $S U(N)$ IM using a local $Z(N)$ invariant potential for $A_{0}$ gauge field. Then, one suggests that the action of confining renormalizable $S U(N)$ model involves a non-polynomial $Z(N)$ periodic term depending on $A_{0}$ gauge field, for instance for $S U(2)$ one uses a potential of the sine-Gordon type. It has been shown 10 that in the weak coupling region $Z(2)$ lattice gauge model coupled to $S O(3)$ spin system with either the standard $S U(2)$ measure or with the sine-Gordon potential could exhibit confinement at zero temperature. This behaviour is in a large extent due to the presence of the measure term which lowers the effective coupling (represented by term tanh $\lambda$ in the ordinary $Z(2)$ model, $\left(\lambda=1 / g^{2}\right)$ ).

A similar situation is expected to happen in finite temperature gauge models in confining disordered phase. This picture is changing in the high temperature deconfinement phase. The loss of confinement implies the measure contribution is either strongly suppress or even completely cancelled. The measure contribution is not able to disorder the system and to keep it in confinement phase. Principally, following scenarios have appeared:

1). The IM term is not essential at all at any energies since the term does not contribute when the dimensional regularization is used. It could mean, that any contribution of the measure in any other regularization is vanishing, after regularization 
is removed [3].

2 ). The IM is completely cancelled by the contribution coming from the integration over space gauge fields. The conclusions drawn by some authors from here concerned the behaviour of the Polyakov loop $L$ and $A_{0}$ condensate in the high temperature phase: $<A_{0}>=0,<L>= \pm 1$ both at one-loop order [1] and at 2-loop order [11.

$3)$. The IM is completely cancelled by the contribution coming from the integration over space gauge fields. This happens both in confinement and in deconfinement phases. For the expectation values we have above critical point $<A_{0}>\neq 0,<L>\neq \pm 1$ (or $<A_{0}>=0$ but $<L>\neq \pm 1$ ). The formal cancellation of the IM in confinement phase does not mean that all factors disordering the system are wiped away from the theory. At very high temperature the transition to the phase with expectation values behaviour as in 1) is possible [12]. Detailed examination of the Debye screening mass 113 showed that this scenario is not in contradiction (at least) with what we expect for the behaviour of the Debye mass.

4). The IM contribution is small but still finite and influences expectation values. Then their behaviour is close to the previous case, but the transition to the phase with vanishing value of condensate and $\langle L\rangle= \pm 1$ is hardly possible.

$5)$. The IM is not cancelled at all and gives an essential contribution to the expectation values at finite energy scale (see, for instance [7]).

Up to now we have been speaking of the naive IM which merely means invariant measure on $S U\left(N_{c}\right)$ gauge group for $A_{0}$ gauge field. One could introduce more general quantity, namely the invariant distribution (ID) of the gauge field configurations as (in the lattice notations)

$$
e^{N V_{I D}}=\int \prod_{x} D \mu\left(L_{x}\right) \delta\left[\sum_{x} L_{x}-N L\right],
$$

where $L_{x}$ is the Polyakov loop and $N$ is a number of lattice sites in a time slice. $D \mu\left(L_{x}\right)$ means the IM on $S U\left(N_{c}\right)$ group and $L$ is the expectation value of the Polyakov loop. The physical meaning of the ID directly follows from its definition: it defines $Z\left(N_{c}\right)$ symmetrical distribution for the expectation value of the Polyakov loop. The same questions concerning the IM could be exposed now for the ID of Polyakov loops. Again, all aforementioned scenarios are proper here in dependence on the features of the ID and the possible cancellation out by integration over space gauge fields.

The goal of the present paper is to investigate which scenario could be realized at finite temperature from the point of view of invariant measure and invariant distribution contributions to the effective potential.

The paper is organized as follows. In section 2 we investigate IM and ID contributions on the lattice in both phases, taking as an example pure $S U(2)$ gauge model. We shall define EP for the $S U(2) \mathrm{PL}$, for the $Z(2) \mathrm{PL}$ and eigenvalues of $S U(2) / Z(2)$ $\mathrm{PL}$ (which can be interpreted as the $A_{0}$ condensate in the continuum theory) with the ID for the PL and calculate their general form. Section 3 is devoted to the discussion of the IM problem in the $S U(2)$ continuum theory. Using results of these two sections we overview aforementioned scenarios and discuss the physical picture of the high temperature phase. 


\section{Invariant measure on the lattice}

To the beginning, let us describe the phase structure of the $(d+1) S U\left(N_{c}\right)$ model at finite temperature. The partition function

$$
Z=\int D \mu\left(U_{n}\right) D \mu\left(U_{0}\right) \exp \left(\lambda \sum_{p} \Omega(\partial p)+\lambda_{0} \sum_{p_{0}} \Omega\left(\partial p_{0}\right)\right),
$$

where $p_{0},(p)$ are time-like (space-like) plaquettes, $\Omega(\partial p)$ is the fundamental plaquette character and

$$
\lambda_{0}=\xi \frac{2 N_{c}}{g^{2}}, \lambda=\xi^{-1} \frac{2 N_{c}}{g^{2}}, \xi=\frac{a_{\sigma}}{a_{t}},
$$

is calculated using the following boundary conditions:

$$
U_{\mu}(x, t)=U_{\mu}\left(x, t+N_{t}\right) .
$$

These conditions (田) generate new physical degrees of freedom which can be taken as the eigenvalues of the Polyakov loop (PL)

$$
W_{x}=P \prod_{t=1}^{N_{t}} U_{0}(x, t)
$$

The compactness in time direction leads to a $Z\left(N_{c}\right)$ global symmetry of the model. This means, multiplication of all links in time direction in a three dimensional $x, y, z$ torus by a $Z\left(N_{c}\right)$ element does not change the action, though a single Polyakov loop transforms as

$$
W_{x} \longrightarrow z W_{x}, z \in Z\left(N_{c}\right) .
$$

Thus, the expectation value of the PL can be used as an order parameter to measure a spontaneous breaking of $Z\left(N_{c}\right)$ symmetry. The corresponding phase transition is well-known as the deconfinement, and in the high temperature phase $Z\left(N_{c}\right)$ symmetry is spontaneously broken [14].

We want to investigate this phase structure from the point of view of the IM contribution to the partition function (2). Presumably, one of the best way to understand IM effects on the dynamics of the gauge system is to consider the effective potential for the PL. This was done for the first time in [ []. The EP $V_{\text {eff }}$ in the strong coupling region of the $S U(2)$ gluodynamics was found in the form

$$
V_{e f f}=2 d \lambda L^{2}+V_{I M},
$$

where $L$ can be interpreted here as an expectation value of the trace of the PL (5) in the fundamental representation and $\lambda \sim\left(\frac{1}{g^{2}}\right)^{N_{t}}\left(N_{t}\right.$ is a number of sites in the time direction). In what follows we use the notation $V_{I M}$ for the IM contribution to the EP. We have then for the $S U(2)$ gauge group

$$
V_{I M}=\ln \left(1-L^{2}\right) .
$$


Since we define the EP with "+" sign, we need to look for its maxima. Analyzing the EP (7), one concludes that at sufficiently small $\lambda$ (low temperatures) the IM term $V_{I M}$ dominates the EP. Maximum is achieved at the point $L=0$, what corresponds to the confinement phase. When $\lambda$ grows, the phase transition takes place to the deconfinement phase with $L \neq 0$ in points of maxima of $V_{\text {eff }}$. We could deduce from this simple example that the IM term might indeed be of great importance, at least in the low temperature phase.

Let us examine this example more carefully. The EP (7) was calculated from the following partition function

$$
Z=\int \prod_{x} d \mu\left(L_{x}\right) \exp \left[\lambda \sum_{x, n} L_{x} L_{x+n}\right]
$$

which can be represented in the obvious parametrization $L_{x}=\cos \phi_{x}$ as

$$
Z=\int \prod_{x} d \phi_{x} \sin ^{2} \phi_{x} \exp \left[\lambda \sum_{x, n} \cos \phi_{x} \cos \phi_{x+n}\right]
$$

It is easy to conclude from the apparent form of the partition function that the very existence of the confinement phase does not depend at all on the presence of the term $\sin ^{2} \phi_{x}$. It is just the ID contribution which is responsible for the vanishing value of the PL at small $\lambda$. Let us illustrate this. We define the EP for the fundamental PL in the original theory (2) as

$$
e^{N V_{e f f}(L)}=\int D \mu\left(U_{n}\right) D \mu\left(L_{x}\right) \exp \left[\lambda \sum_{p} \Omega(\partial p)+\lambda_{0} \sum_{p_{0}} \Omega\left(\partial p_{0}\right)\right] \delta\left[\sum_{x} L_{x}-N L\right] .
$$

For the effective theory (10) it gives

$$
e^{N V_{e f f}(L)}=\int \prod_{x} d \phi_{x} \sin ^{2} \phi_{x} \exp \left[\lambda \sum_{x, n} \cos \phi_{x} \cos \phi_{x+n}\right] \delta\left[\sum_{x} \cos \phi_{x}-N L\right] .
$$

Applying now the mean-spin approximation for the right-hand side of the last equation we arrive to the EP of the form

$$
V_{e f f}(L)=2 d \lambda L^{2}+V_{I D}
$$

with $V_{I D}$ defined as

$$
V_{I D}=\frac{1}{N} \ln \int \prod_{x} d \phi_{x} \sin ^{2} \phi_{x} \delta\left[\sum_{x} \cos \phi_{x}-N L\right] .
$$

Analyzing the EP (13) one can convince himself that the presence of the measure term $\sin ^{2} \phi_{x}$ is not so important for the phase structure of the theory: at small $\lambda$ the PL is vanishing even if we neglect this term. Certainly, its presence reveals some specific features of $S U(2)$ theory but the whole contribution of the ID is much more essential. 
There are certain regions for the PL where the asymptotes of the ID and the IM are qualitatively the same. We shall return to the discussion about the asymptotic behaviour later. One of the main goals of the following discussion is to show that despite the fact that the cancellation of the IM takes place, the ID cannot be cancelled by any integration over space gauge fields. If this contribution had been correctly taken into account in the perturbative calculation one would hope to find a way to calculate a reliable $\mathrm{EP}$ for the $\mathrm{PL}$ in the continuum theory.

We are ready now to investigate the problem of the cancellation of IM and ID in $S U(2)$ theory. First of all, we consider the chromoelectric part of the action in the Hamiltonian formulation of LGT. The effects of the magnetic part can be easier seen in the Euclidean version.

The Hamiltonian of the lattice gluodynamics in the strong coupling approximation includes only the chromoelectric part

$$
H=\sum_{\text {links }}\left(\frac{g^{2}}{2 a}\right) E^{2}(l)
$$

where $E(l)=i \partial / \partial\left(A_{l}\right)$ are the chromoelectric field operators. In this approach the chromomagnetic term can be treated perturbatively at $g^{2} \rightarrow \infty$. The calculation of the partition function

$$
Z=\tilde{S p} \exp (-\beta H)
$$

is connected with the summation over local gauge-invariant states. This is reflected by the symbol $\tilde{S p}$ in (16). $\beta$ is the inverse temperature. The corresponding physical Hilbert space is determined by Gauss' law. By conventional procedure one gets the partition function of the form (see, for instance, [5])

$$
Z=\int \prod_{x} d \mu\left(\phi_{x}\right) \prod_{x, n}\left[\sum_{l=0, \frac{1}{2}, \ldots} e^{-\gamma C_{l}} \Omega_{l}\left(\phi_{x}\right) \Omega_{l}\left(\phi_{x+n}\right)\right] .
$$

$C_{l}$ is here the quadratic Casimir operator, $\gamma=\frac{\beta g^{2}}{2 a}$. $\Omega_{l}$ is the character of $l$-th irreducible representation of the $S U(2)$ group. The fundamental character $\Omega_{1 / 2}$ represents the PL in this formulation. We have shown in [5] that the same partition function can be obtained in the Euclidean version of the theory restricted to time-like plaquettes after integrating out space gauge fields. Thus, we can study the problem of our interest in this model. Notice, that the invariant measure $d \mu\left(\phi_{x}\right)$ appeared after representation of Gauss' law delta-function on the $S U(2)$ group. This remark is rather important since the IM in the approach of Ref. [3] has the same origin.

The formal cancellation of the IM can be seen from the last equation. Substituting for $S U(2)$ characters the following representation

$$
\Omega_{l}(\phi)=\frac{\sin (2 l+1) \phi}{\sin \phi},
$$

into Eq.(17) we find that the measure term $\sin ^{2} \phi$ is cancelled by the denominator of the product of characters in each space point. (Expanding product $\prod_{x, n}$ in (17) over 
closed graphs one sees the exact cancellation of the measure with the denominator of the character in every point which enters one time into graph. If some point $x$ enters more than once in the graph we have $\left(\sin ^{2} \phi\right)^{n-1}$ in the denominator where $n$ is a number of times when graph passes through point $x$ ).

We propose to compare now two effective potentials. The first one includes only the invariant measure whereas the second one reflects the presence of the invariant distribution.

For the naive effective potential (which is analogy of the EP in (17)) summing up over all representations of the group (up to a constant independent of $\phi$ ) we got

$$
V_{e f f}(\phi)=2 d \ln \left[1-\frac{\Theta_{2}\left(e^{-\gamma}, 2 \phi\right)+\Theta_{3}\left(e^{-\gamma}, 2 \phi\right)}{\Theta_{2}\left(e^{-\gamma}, 0\right)+\Theta_{3}\left(e^{-\gamma}, 0\right)}\right]-2 d \ln \sin ^{2} \phi+V_{I M}(\phi),
$$

where $\Theta_{i}$ is the Jacobi theta-function. The fundamental Polyakov loop is $L=\cos \phi$ in this representation and $V_{I M}=\ln \sin ^{2} \phi$. Although this EP has a more complicated analytical structure than the one presented in (7), it possesses the same fundamental features. Namely, at low temperatures $(\gamma \rightarrow \infty)$ it has the maximum at $\phi=\pi / 2$ which corresponds to the vanishing mean value of the PL. The presence of $V_{I M}$ is crucial here: if we had neglected its contribution we would find the only phase with $\phi=0, \pi$ corresponding to $Z(2)$ broken deconfinement phase. Thus, this cancellation has a rather formal character in the presented picture: The invariant measure carries a memory of the invariant distribution of the Polyakov loop in the naive effective potential and as such cannot be cancelled (at the same time the IM cancels the important part of the potential gluonic energy).

For the EP with the invariant distribution term we should substitute, following the definition (11), $V_{I D}$ from (11) into (19). Assuming the cancellation of the measure we come to

$$
V_{e f f}(\phi)=2 d \ln \left[1-\frac{\Theta_{2}\left(e^{-\gamma}, 2 \phi\right)+\Theta_{3}\left(e^{-\gamma}, 2 \phi\right)}{\Theta_{2}\left(e^{-\gamma}, 0\right)+\Theta_{3}\left(e^{-\gamma}, 0\right)}\right]-(2 d-1) \ln \sin ^{2} \phi+V_{I D}(L) .
$$

$V_{I D}(L)$ coincides with (14), where the IM should be omitted

$$
V_{I D}=\frac{1}{N} \ln \int \prod_{x} d \phi_{x} \delta\left[\sum_{x} \cos \phi_{x}-N L\right]
$$

Numerical investigation reveals the same fundamental features as those described above for the naive EP. Moreover, let us suppose that we neglected the measure contribution entirely, i.e.

$$
(2 d-1) \ln \sin ^{2} \phi \rightarrow 2 d \ln \sin ^{2} \phi
$$

The qualitative picture is still the same because $V_{I D}$ as defined in (21) gives dominating $Z(2)$ symmetrical distribution of the $\mathrm{PL}$ and possesses a maximum at $\phi=\pi / 2$. These features are quite understandable because at $L \ll 1$ we have from (21) $V_{I D} \approx-L^{2}$ which coincides qualitatively with the corresponding behaviour of $V_{I D}$ as defined in (14) and with the behaviour of the IM in the same region of $L$. 
What can we learn from these examples? The invariant measure represents in the naive EP a contribution of more general quality, namely the invariant distribution of the Polyakov loop. Having the maximum at the vanishing value of the $\mathrm{PL}$, the invariant distribution forces the system to stay in the confinement phase at low temperatures. As temperature increases the potential energy dominates the EP and the system undergoes deconfining transition. Above the critical point the ID also contributes to the EP. This implies that $\phi \neq 0, \pi$ and, consequently, the $\mathrm{PL}$ is not equal \pm 1 , at least close to the critical temperature. In the presented approximation we have not found any terms which could potentially cancel the ID term at high temperature (it is obvious that such a cancellation is impossible in confinement phase). One would stress that, because this approach is the strong coupling approximation to the problem, this cancellation could take place in the region of the weak coupling of the continuum theory. This possibility cannot be excluded a priori so we look at this problem in the next section.

We would like now to investigate effects of the magnetic term on this strong coupling picture to understand what happens with IM and ID contributions at arbitrary coupling constant. We consider the Euclidean version of $S U(2)$ model with partition function (2). We fix a gauge where all static $U_{0}$ matrices are placed between $N_{t}$-th and $N_{t}+1=$ $N_{1}$-th sites of periodic lattice, grouping in the PL $W_{x}$ (before taking the trace). $W_{x}$ can now be taken in the diagonal form. This gauge is of special interest because the Faddeev-Popov determinant in this gauge coincides with the group integration measure for the PL [8]. Using the definition (11) we can put down the effective potential in a more general form as

$$
e^{N\left[V_{e f f}(L)-V(T=0)\right]}=\int D \mu\left(L_{x}\right) \exp \left[S_{e f f}\left(L_{x}\right)\right] \delta\left[\sum_{x} L_{x}-N L\right],
$$

where we subtract the contribution to $V_{\text {eff }}$ at zero temperature. Since we have the compact group integration over space gauge matrices we do not fix a gauge for them. In this way we can observe a gauge independent cancellation of the IM. In the fixed gauge we have for $S_{\text {eff }}$ on symmetrical lattice $(\xi=1)$ expanding the plaquette action into series over irreducible representations $l$ the relation:

$$
\begin{array}{r}
e^{S_{e f f}\left(L_{x}=\operatorname{Tr} W_{x}\right)}=\operatorname{const}(\lambda) \int D \mu\left(U_{n}\right) \prod_{p}\left[\sum_{l} K_{l}(\lambda) \Omega_{l}(\partial p)\right] \\
\prod_{x, n}\left[\prod_{t=1}^{N_{t}-1} \sum_{l} K_{l}(\lambda)\left(\operatorname{Tr} U_{n}(t) U_{n}^{+}(t+1)\right)_{l}\right] \\
\prod_{x, n}\left[\sum_{l} K_{l}(\lambda)\left(\operatorname{Tr} W_{x} U_{n}\left(N_{t}\right) W_{x+n}^{+} U_{n}^{+}(t=1)\right)_{l}\right] .
\end{array}
$$

$\Omega_{l}$ is the character of $l$-th representation of the space-like plaquette and $K_{l}(\lambda)$ are the known coefficients of the character expansion. The second and the third line in (23) represent contribution of time-like plaquettes. In this approach the theory was investigated in [15]. 
Two properties of the invariant $S U(2)$ integration are essential here: 1) only closed surfaces contribute to $\left.S_{e f f} ; 2\right) S_{\text {eff }}$ is a functional of $L_{x}$ because this is the only gauge invariant configuration after we have integrated out space gauge fields. Let $G$ be an arbitrary graph in $d$ space dimensions. Performing the invariant integration in (23) we find the effective theory of the PL expressed in form of a sum over all possible graphs in $d$ dimensions

$$
e^{S_{e f f}\left(L_{x}\right)}=\operatorname{const}(\lambda) \sum_{G} \sum_{r_{l}} \prod_{l \in G}\left[L_{r_{l}}(x) L_{r_{l}}(x+n)\right] C^{G}\left(r_{1}, \ldots, r_{G}\right),
$$

$l$ is a link belonging to the graph $\mathrm{G}$. Every link can carry its own representation $r_{l}$. It is an enormous task to calculate an apparent form of the coefficients of this expansion. For instance, in the simplest case when every link which we have integrate over in (23) enters only twice to the closed surface, we have

$$
C^{G}\left(r_{1}, \ldots, r_{G}\right)=\sum_{S} K_{r}^{|S|} N(S)
$$

Because of the invariant integration every plaquette on a surface carries the same representation $r$. $S$ is a closed surface which forms a closed graph (or a part of a closed graph if it is a product of different closed paths) $\mathrm{G}$ in the time-slice between points $N_{t}$ and $N_{t}+1$. $|S|$ is the full number of plaquettes on such a surface and $N(S)$ is number of surfaces with $|S|$ plaquettes contributing to the graph $G$. An example of surface of the form (25) one finds if we consider time-like plaquettes. A surface which is going around the lattice and is built only from time-like plaquettes cannot be expanded in space direction and we have from (25) $C^{G}(r)=K_{r}^{N_{t}}$ (see, for instance, [15]). An arbitrary $C^{G}$ has a similar structure but every plaquette on the surface may carry it own representation with the restriction coming from invariant integration over a link which is common for more then two plaquettes. A surface must go around the whole lattice to include $L_{x}$. Otherwise, the contribution of a surface is simply constant because the result of the invariant integration does not depend on the gauge field $U_{0}$. This reflects the known fact that in the theory without periodic boundary conditions we can always choose the gauge $U_{0}=1$. To get an $L_{x}$ dependence we have to obtain a gauge invariant loop after the integration and the only one available is the loop wrapping the lattice in the time direction. Fortunately, for our goal it is not necessary to have an explicit form of these coefficients. Calculating $V(T=0)$ we find that on the symmetric lattice this potential coincides with $S_{\text {eff }}\left(L_{x}=0\right)$. This contribution enters $e^{S_{e f f}\left(L_{x}\right)}$ when $G$ is a trivial nil graph and includes the summation over all closed surfaces independent of $L_{x}$. Dividing by this term we have a renormalization of coefficients $C^{G}$. Keeping old notation for new coefficients we end up with the following EP

$$
\begin{array}{r}
e^{N\left[V_{e f f}(L)\right]}=\sum_{G} \sum_{r_{l}} C^{G}\left(r_{1}, \ldots, r_{G}\right) \\
\int D \mu\left(L_{x}\right) \delta\left[\sum_{x} L_{x}-N L\right] \prod_{l \in G}\left[L_{r_{l}}(x) L_{r_{l}}(x+n)\right] .
\end{array}
$$


Two facts immediately appears from this representation of the EP. The first one concerns the static gauge: its fixing was not essential at all in obtaining Eq.(26). The EP is expressed through gauge invariant quantity $L_{x}$ and is gauge independent. Without gauge fixing we would get $L_{x}$ as defined in (5). Because of the invariant integration we may omit all the integrations on the time-like links except the last one. To get a diagonal form of the PL we should now use Weyl's representation for the measure and for $S U(2)$ matrices. Non-diagonal matrices do not contribute to the trace of $W_{x}$. The IM for the diagonal part in Weyl's representation coincides with the Faddeev-Popov determinant and we can study the problem in this approach, too. This procedure leads again to the EP (26). On this basis we expect that the static gauge will be equally good in the continuum theory discussed further.

The second fact is the cancellation of the IM which happens here in the same manner as in the strong coupling regime of the Hamiltonian formulation described after Eq.(18). We want now to calculate a general form of the EP for $Z(2) \mathrm{PL}$. We introduce the following quantity

$$
F_{G}(L)=\int D \mu\left(L_{x}\right) \delta\left[\sum_{x} L_{x}-N L\right] \prod_{l \in G}\left[L_{r_{l}}(x) L_{r_{l}}(x+n)\right] .
$$

To get the EP for the $Z(2) \mathrm{PL}$ we have to substitute the following delta function

$$
\delta\left[\sum_{x} s_{x}-N s\right]
$$

into the last equation. $s_{x}= \pm 1$ is the Ising spin. We should use the representation for the PL in (27)

$$
L_{r_{l}}(x)=s_{x}^{2 r_{l}} \bar{L}_{r_{l}}(x),
$$

where $\bar{L}_{r_{l}}(x) \in S U(2) / Z(2)$ together with the corresponding representation for the measure

$$
\int d \mu\left(L_{x}\right)=\frac{1}{2 \pi} \sum_{s= \pm 1} \int_{-\pi / 2}^{\pi / 2} \sin ^{2} \phi d \phi .
$$

We adjust the following approximation for the integration over $S U(2) / Z(2)$ part of the group: $\phi=\phi_{0}=$ const. This is in the spirit of Ref. [16]. We are not supposed to calculate $\phi_{0}$ from the analogy with the Ising model but rather from an independent minimization procedure. In this case, the constant $\phi_{0}$ may be interpreted as the $A_{0}$ condensate whose only nontrivial values lie in the $S U(2) / Z(2)$ subgroup. Let $n_{0}(G)$ be the number of points $x$ in the graph $G \cdot n_{0}(G) \neq|G|$, if more than two links enter any point in the graph. $n_{1}(G)$ is a number of points $x$ in the graph $G$ in which $\sum_{l} r_{l}=\frac{2 k+1}{2}$, where $l$ is a link entering the point $x$. Then, the result of the calculation of the function $F_{G}(L)$ in the limit $N \rightarrow \infty$ and for sufficiently small $s$ can be expressed as

$$
\begin{aligned}
F_{G}\left(s, \phi_{0}\right)= & \exp \left[-\frac{N^{2} s^{2}}{2\left(N-n_{1}(G)\right)}+\left(N-n_{0}(G)\right) \log \sin ^{2} \phi_{0}\right] \\
& \prod_{l \in G} \sin ^{2}\left(2 r_{l}+1\right) \phi_{0} H_{n_{1}(G)}\left(\frac{N s}{\left(2\left(N-n_{1}(G)\right)\right)^{1 / 2}}\right),
\end{aligned}
$$


where $H_{n}(z)$ is the Hermite polynomial of the n-th order. $\phi_{0}$ can be calculated from the following effective potential

$$
\begin{array}{r}
e^{N\left[V_{\text {eff }}\left(\phi_{0}\right)\right]}=\sum_{G^{\prime}} \sum_{r_{l}} C^{G^{\prime}}\left(r_{1}, \ldots, r_{G^{\prime}}\right) \\
\exp \left[\left(N-n_{0}(G)\right) \log \sin ^{2} \phi_{0}\right] \prod_{l \in G} \sin ^{2}\left(2 r_{l}+1\right) \phi_{0} .
\end{array}
$$

$\sum_{G^{\prime}}$ is the sum over loops in which every point may enter only even number of times. What may we conclude from these general representations for EP? Some general properties can be seen without knowing the exact form of the coefficients $C^{G}$. For sufficiently small $L$ the ID of the PL (11) is:

$$
V_{I D}=-\frac{L^{2}}{2} .
$$

This contribution (for $Z(2) \mathrm{PL}$ ) is contained in (28) when $G$ is a trivial graph. This is the only contribution which tends to disorder a system although other contributions presented by $H_{k}(s b)$ are increasing functions of $s$. There always exists a small coupling $\lambda$ such as the contribution coming from the sum over $G$ is small and the ID term is dominating the EP. In this case we have the maximum of the EP at $s=0$. Therefore, the full $S U(2) \mathrm{PL}$ equals zero as well in the force of the inequality

$$
\frac{1}{2} \operatorname{Tr} W_{x} \leq s_{x}
$$

The presence of the term $\log \sin ^{2} \phi$ is not crucial at all when a vanishing value for the PL is achieved. Let us discuss now the fate of the $A_{0}$ condensate as it follows from (29). The EP would achieve its maximum at $\phi=0$ for any coupling constant $\lambda$ had we neglected the contribution $\log \sin ^{2} \phi_{0}$. This is in a full accordance with Ref. [3] where the absence of the condensate has been claimed in case if we omit the IM from the partition function. Our formulae demonstrate something different, that we are not allowed to simply omit the measure, at least in the lattice regularization. It unambiguously follows from (29) that if the invariant measure $N \log \sin ^{2} \phi_{0}$ is present in the effective action, $\phi_{0}$ always differs from zero. The values $\pm \frac{\pi}{2}$ are trivial and they are achieved in the confinement region (though it is difficult to prove this rigorously). Starting from deconfinement critical temperature, $\phi_{0}$ goes away from the edge of the integration region forming a saddle point configuration in $S U(2) / Z(2)$ subgroup. In the continuum limit this saddle point could be interpreted as the $A_{0}$ condensate. Thus, there is no doubt that the condensate exists on the lattice. Another argument, supporting this conclusion, follows from the universality and will be discussed elsewhere. The central question, whether this nontrivial saddle point survives the transition to the continuum limit, is obviously a nontrivial problem (see, for the discussion, in [7]). We shall return to this problem in the next publication. 


\section{Invariant measure in the continuum}

The lattice consideration provided us with some picture as of the invariant measure and the invariant distribution behaviour in the quantum theory. To find out IM properties and their influence on the phase diagram of the gauge theory in the continuum spacetime is a more difficult question. Some discussion of this point can be found in [7]. We shall overview this discussion in the Summary. To specify the problem and to be as close to the lattice picture as possible in sense of the interpretation of results we fix a static gauge

$$
A_{0}^{a}(x, t)=\delta^{3, a} A_{0}^{a}(x) .
$$

We recall briefly how the IM term appears in the chosen gauge as the Faddeev-Popov determinant. The partition function for the finite temperature Euclidean theory reads 117

$$
Z(\beta)=\mathcal{N} \int\left[\mathcal{D} A_{\mu}^{a}(x, t)\right] \Delta_{F P}\left[f\left(A_{\mu}\right)\right] \delta\left[f\left(A_{\mu}\right)\right] \exp \left[-S_{e}\right],
$$

where $S_{e}$ is Euclidean action, $f\left(A_{\mu}\right)$ defines the gauge fixing condition and integrals are calculated over all gauge fields $A_{\mu}^{a}(x, t)$ obeying the periodicity conditions $A_{\mu}^{a}(x, t)=$ $A_{\mu}^{a}(x, t+\beta)$. The Faddeev-Popov determinant $\Delta_{F P}$ is defined by the group integration as

$$
\Delta_{F P} \int[\mathcal{D} U] \delta\left[f\left(A^{U}\right)\right]=1, \quad U=\exp \left[-i \omega_{i}^{a}(x, t) \frac{\sigma_{a}}{2}\right],
$$

where $A^{U}$ denotes the $U$ - transform of the field $A$. We calculate the Faddeev-Popov determinant by the standard prescription exploiting the appearance of the delta functional in Eq.(32). We only need to know the Faddeev-Popov determinant for gauge fields $A_{\mu}^{a}$ transformed by $U$ near identity. The gauge fixing condition (31) determines the form of the determinant. Because in the non-perturbatively defined theory this gauge has been shown as reliable, we expect that it is also good in the continuum theory. In this gauge quantum fluctuations around the classical $A_{0}$ field are both static and in direction which commutes with the classical field. We introduce new integration variables $\omega^{a}(x, t)$ into the integral in Eq.(33) by Taylor expansion of transformation matrices $U$. We rewrite also the gauge fixing condition $f\left[A^{U}\right]$ for an infinitesimal gauge transformation and the Faddeev-Popov determinant after the Fourier transform becomes

$$
\Delta_{F P}^{-1}=\int\left[\mathcal{D} \tilde{\omega}^{a}(x, n)\right] \delta\left[\left(\frac{-2 \pi n i}{g \beta} \delta^{a, b}+\varepsilon^{a b 3} A_{0}^{3}(x)\right) \tilde{\omega}^{b}(x, n)\right] .
$$

We have found that the functional integral of the zero-th Fourier mode should be treated separately. The result for $n=0$ integration over $\left[\mathcal{D} \tilde{\omega}^{a}(x, n=0)\right]$ fields is $\mathcal{N} \operatorname{Det}^{-1}\left[\left(A_{0}^{3}(x)\right)^{2}\right]$, where $\mathcal{N}$ corresponds to the space volume obtained for $a=3$ integration. Skipping out details of the calculation of functional determinants for 
nonzero Fourier modes, the Faddeev-Popov determinant contribution to the effective action is the periodic function in the sense of the transformation

$$
\frac{g \beta A_{0}^{3}(x)}{2 \pi} \rightarrow \frac{g \beta A_{0}^{3}(x)}{2 \pi}+n_{0}
$$

where $n_{0}$ is an arbitrary integer. In the notation

$$
X(x)=\left.\frac{g \beta A_{0}^{3}(x)}{2 \pi}\right|_{\bmod 1}
$$

the Faddeev-Popov determinant has the form

$$
\Delta_{F P}=\exp \left[\int \frac{d^{3} k}{(2 \pi)^{3}} \int d^{3} x\{\ln (g \beta)+2[\ln 2 \sin (\pi X(x))]\}\right],
$$

which coincides with the IM term up to the constant $\ln (g \beta)$. The appearance of this constant is an important secondary result, automatically ensuring the change of the integration measure in Eq.(32) as

$$
\left[\mathcal{D} A_{0}^{3}(x)\right] \rightarrow\left[\mathcal{D}\left(g \beta A_{0}^{3}(x)\right)\right]
$$

In the continuum one works with terms in the background field decomposition of the IM, when the $A_{0}^{3}(x)$ field is supposed to have the form

$$
A_{0}^{3}(x)=A_{0}+a_{0}^{3}(x),
$$

where $A_{0}$ is the classical constant field and $a_{0}^{3}(x)$ are quantum fluctuations of the field. The discussion in previous investigations [1, 2, 6] concerned the cancellation of terms with different powers of $a_{0}^{3}(x)$ in the background field expansion of the IM (37) by terms of the effective action which appear due to the functional integration over space fields. There is no doubt that the zero-th and first order terms in power of the field $a_{0}^{3}(x)$ cancel, the ambiguity concerns the second order term. In the following we are going to present the calculation without background field decomposition (38). We shall show that the IM can be cancelled by the functional determinant due to the integration over space fields. On the other side, we adduce an example when the noncomplete integration over space fields only modifies the IM.

In the fixed gauge the $S U(2)$ Euclidean action at finite temperature has the form

$$
\begin{aligned}
S_{e}= & \frac{1}{4} \int_{0}^{\beta} d t \int d^{3} x F_{\mu \nu}^{a} F_{\mu \nu=}^{a}= \\
& \frac{1}{2} \int_{0}^{\beta} d t \int d^{3} x\left\{\left(\partial_{i} A_{0}^{3}(x)\right)^{2}+\left(\partial_{0} A_{i}^{a}(x, t)\right)^{2}-2 g \varepsilon^{a 3 c}\left(\partial_{0} A_{i}^{a}(x, t)\right) A_{0}^{3}(x) A_{i}^{c}(x, t)\right. \\
& +\left(g A_{0}^{3}(x)\right)^{2}\left[\left(A_{i}^{1}(x, t)\right)^{2}+\left(A_{i}^{2}(x, t)\right)^{2}\right]+\frac{1}{2}\left(\partial_{i} A_{k}^{a}(x, t)+\partial_{k} A_{i}^{a}(x, t)\right)^{2}- \\
& 2 g \varepsilon^{a b c}\left(\partial_{i} A_{k}^{a}(x, t)\right) A_{i}^{b}(x, t) A_{k}^{c}(x, t)+ \\
& \left.\frac{g^{2}}{2}\left(A_{i}^{b}(x, t) A_{k}^{c}(x, t) A_{i}^{b}(x, t) A_{k}^{c}(x, t)-A_{i}^{b}(x, t) A_{k}^{c}(x, t) A_{i}^{c}(x, t) A_{k}^{b}(x, t)\right)\right\} .
\end{aligned}
$$


In the following we are going to neglect the terms of the third and fourth order in gauge potentials $A_{i}^{a}(x, t)$. It has been shown [2] that integrating out fields $A_{i}^{1}(x, t), A_{i}^{2}(x, t)$ completely (i.e. zero Fourier modes also) from the action (39), one obtains the effective theory with the periodic effective potential in the variable $\frac{g \beta A_{0}}{2 \pi}$, with the nontrivial minima for $A_{0}$. Infrared stability of the functional integration over fields is assured by the "nonzero mass term" squared in Eq.(39) for potentials $A_{i}^{1}(x, t), A_{i}^{2}(x, t)$ with the mass proportional to $\left|g A_{0}^{3}(x)\right|$. For nonzero Fourier modes of space gauge potentials the rôle of mass terms in the Appelquist - Carazzone decoupling mechanism[18] are played by Matsubara frequencies. It seems natural to demand the same magnitude of the mass term for all fields maintained by the Appelquist - Carazzone mechanism. Therefore, we suppose that the effective theory appearing after integrating out space gauge potentials is reasonable, if the ratio of the mass term for the zero-order field and first Matsubara frequency is non-negligible, for instance

$$
\frac{\left(g \beta A_{0}\right)}{2 \pi}>\exp (-1) \text {. }
$$

The opposite case will be studied later. The result of the integration is effective static theory with fields $A_{0}^{3}(x)$ and $\mathcal{A}_{i}^{3}(x, n=0)$, the zero-th Fourier component of the gauge potential $A_{i}^{3}(x, t)$ as dynamical degrees of freedom. The details of calculations can be found in [2], here we give a sketch of the results. The nonlocal determinant obtained by the aforementioned integration has the form in the zeta function regularization scheme 19

$$
\operatorname{Det}^{-\frac{1}{2}}[\mathcal{M}+\mathcal{L}+\mathcal{V}]=\exp \left\{\frac{1}{2} \lim _{s \rightarrow 0} \frac{\partial}{\partial s} \frac{1}{\Gamma(s)} \int_{0}^{\infty} t^{s-1} d t \operatorname{Tr}\left[e^{-(\mathcal{M}+\mathcal{L}+\mathcal{V}) t}\right]\right\} .
$$

$\mathcal{M}, \mathcal{L}, \mathcal{V}$ are the operators, derived from Eq.(39). The exponential operator can be expressed term-by-term using local Schwinger operator decomposition [20]. Only the zero-th order term of such decomposition does not contain an interaction term of fields $A_{0}^{3}(x)$ and $\mathcal{A}_{i}^{3}(x, n=0)$ and, therefore it may have the common features with the invariant measure (37). The zero-th order term of (41) has the following form

$$
\operatorname{Det}_{0}^{-\frac{1}{2}}=\exp \left\{\frac{1}{2} \lim _{s \rightarrow 0} \frac{\partial}{\partial s} \frac{1}{\Gamma(s)} \int_{0}^{\infty} t^{s-1} d t \operatorname{Tr}\left[e^{-(\mathcal{M}+\mathcal{L}) t}\right]\right\} .
$$

The operator $\mathcal{M}$ is a $6 \times 6$ matrix operator, diagonal in $\mid n, p>$ representation and the operator $\mathcal{L}$ is also a $6 \times 6$ matrix operator diagonal in $\mid x>$ representation. For the functional trace operator expressed in the $p$-representation we use the definition

$$
\operatorname{Tr} \mathcal{O}=\sum_{n=-\infty}^{\infty} \int \frac{d^{3} p}{(2 \pi)^{3}} \operatorname{tr}<p, n|\mathcal{O}| p, n>
$$

We are going to calculate the term

$$
\operatorname{Tr} e^{-(\mathcal{M}+\mathcal{L}) t}=\int \frac{d^{3} p}{(2 \pi)^{3}} \sum_{n=-\infty}^{+\infty}<p, n\left|\left\{\sum_{k=0}^{+\infty} \frac{(-t)^{k}}{k !} \operatorname{tr}(\mathcal{M}+\mathcal{L})^{k}\right\}\right| p, n>,
$$


where $t r$ stands for the trace over matrix. The key step is the calculation of the term

$$
\operatorname{tr}<p, n\left|(\mathcal{M}+\mathcal{L})^{k}\right| p, n>=\operatorname{tr}\{<p, n|(\mathcal{M}+\mathcal{L}) \ldots . .(\mathcal{M}+\mathcal{L})| p, n>\} .
$$

The last relation includes $2^{k}$ different terms. The operator $\mathcal{L}$ appears $\nu$-times in $\left(\begin{array}{l}\nu \\ k\end{array}\right)$ terms. Our goal is to arrange in each term of Eq.(44) the successive group of $\mathcal{L}$ operators. The advantage of this step follows from practical reasons of product trace calculations of operators diagonal in $p$-, or $x$-representations. Introducing the commutation relation

$$
[\mathcal{M}, \mathcal{L}]=\mathcal{M L}-\mathcal{L} \mathcal{M}
$$

we get from Eq.(44)

$$
\operatorname{tr}<p, n\left|(\mathcal{M}+\mathcal{L})^{k}\right| p, n>=\sum_{\nu=0}^{k}\left(\begin{array}{l}
\nu \\
k
\end{array}\right) \operatorname{tr}<p, n\left|\mathcal{M}^{k-\nu} \mathcal{L}^{\nu}\right| p, n>+\mathcal{T}(k) .
$$

$\mathcal{T}(k)$ is the term appearing due to commutations of the $\mathcal{M}$ and $\mathcal{L}$ operators. $\mathcal{T}(k)$ is composed of the trace of the product of operators $\mathcal{M}, \mathcal{L}$ and their commutators. For example, for $k=4$ we have

$$
\mathcal{T}(4)=2 \operatorname{tr}\{\langle p, n|\mathcal{M L}[\mathcal{M}, \mathcal{L}]| p, n>\} .
$$

We do not discuss the terms $\mathcal{T}(k)$ here, because they are not important for the calculation of the term cancelling the IM.

When we replace corresponding expressions in Eq.(43) by Eq.(46), we obtain

$$
\begin{gathered}
\operatorname{Tr} \exp \{-(\mathcal{M}+\mathcal{L}) t\}=\int d^{3} x \int \frac{d^{3} p}{(2 \pi)^{3}} \sum_{n=-\infty}^{+\infty} \\
\sum_{k=0}^{+\infty} \frac{(-t)^{k}}{k !}\left\{\sum_{\nu=0}^{k}\left(\begin{array}{l}
\nu \\
k
\end{array}\right) \operatorname{tr}\left[\mathcal{M}^{k-\nu}(p, n) \mathcal{L}^{\nu}(x)\right]+\mathcal{T}(k)\right\} .
\end{gathered}
$$

In the last expression $\mathcal{M}(p, n)$ and $\mathcal{L}(x)$ are $c$-number matrices

$$
\begin{aligned}
& \mathcal{M}^{k-\nu}(p, n)=\left|\begin{array}{cc}
M^{k-\nu} & 0 \\
0 & M^{k-\nu}
\end{array}\right|, \\
& \mathcal{L}^{\nu}(x)=\left|\begin{array}{cc}
\frac{1}{2}\left\{\left(V_{1}+V_{2}\right)^{\nu}+\left(V_{1}-V_{2}\right)^{\nu}\right\} & -\frac{i}{2}\left\{\left(V_{1}+V_{2}\right)^{\nu}-\left(V_{1}-V_{2}\right)^{\nu}\right\} \\
\frac{i}{2}\left\{\left(V_{1}+V_{2}\right)^{\nu}-\left(V_{1}-V_{2}\right)^{\nu}\right\} & \frac{1}{2}\left\{\left(V_{1}+V_{2}\right)^{\nu}+\left(V_{1}-V_{2}\right)^{\nu}\right\}
\end{array}\right|, \\
& M_{i j}^{k-\nu}=\left[\left(\frac{2 \pi n}{\beta}\right)^{2}+p^{2}\right]^{k-\nu}\left(\delta_{i j}-\frac{p_{i} p_{j}}{p^{2}}\right)+\frac{p_{i} p_{j}}{p^{2}}\left[\left(\frac{2 \pi n}{\beta}\right)^{2}\right]^{k-\nu}, \\
& \left(V_{1}\right)_{i j}(x)=\left(g A_{0}^{3}(x)\right)^{2} \delta_{i j}, \\
& \left(V_{2}\right)_{i j}(x)=2 \frac{2 \pi n}{\beta} g A_{0}^{3}(x) \delta_{i j} \text {. }
\end{aligned}
$$


After $t r$ operation over $3 \times 3$ matrices and summation over indices $\nu, k$ in Eq.(48), we have finally for Eq.(43), excluding the terms containing $\mathcal{T}(k)$

$$
\begin{aligned}
& \operatorname{Tr} \exp \{-(\mathcal{M}+\mathcal{L}) t\}=\int d^{3} x \int \frac{d^{3} p}{(2 \pi)^{3}} \sum_{n=-\infty}^{+\infty} \\
& \left\{4 e^{-\left[\left(\frac{2 \pi n}{\beta}+g A_{0}^{3}(x)\right)^{2}+p^{2}\right] t}+2 e^{-\left[\left(\frac{2 \pi n}{\beta}+g A_{0}^{3}(x)\right)^{2}\right] t}\right\} .
\end{aligned}
$$

Inserting this expression into Eq.(42), we can proceed by the Melin transform

$$
\begin{aligned}
\int_{0}^{\infty} t^{s-1} \operatorname{Tr} e^{-(\mathcal{M}+\mathcal{L}) t} d t & = \\
& \Gamma(s) \int d^{3} x \int \frac{d^{3} p}{(2 \pi)^{3}} \sum_{n=-\infty}^{n=+\infty}\left\{\frac{4}{\left[\left(\frac{2 \pi n}{\beta}+g A_{0}^{3}(x)\right)^{2}+p^{2}\right]^{s}}+\frac{2}{\left[\left(\frac{2 \pi n}{\beta}+g A_{0}^{3}(x)\right)^{2}\right]^{s}}\right\} .
\end{aligned}
$$

In the last equation the periodicity (35) of this contribution to effective action holds. The proof of the periodicity of the effective potential as well as the corresponding effective action follows from the preceding feature of functional determinant contributions. Let us stress that if the summation index $n$ is not going over full range $(-\infty,+\infty)$, then contributions like Eq.(51) are not periodic in the $A_{0}$ gauge field. We are confronted with such a situation in the case when all zero Fourier modes are dynamical variables of the theory. Then, the summation over contributions to the effective potential does not contain terms with $n=0$ and the periodicity is lost.

We follow the definition of Riemann's zeta function

$$
\sum_{n=0}^{\infty} \frac{1}{(n+a)^{s}}=\zeta(s, a)
$$

to evaluate the second term in the right-hand side of Eq.(51). In the notation (36) we find

$$
\begin{array}{r}
\left(\frac{1}{2} \lim _{s \rightarrow 0} \frac{\partial}{\partial s} \frac{1}{\Gamma(s)}\right) \Gamma(s) \int d^{3} x \int \frac{d^{3} p}{(2 \pi)^{3}} \sum_{n=-\infty}^{+\infty} \frac{2}{\left[\left(\frac{2 \pi n}{\beta}+g A_{0}^{3}(x)\right)^{2}\right]^{s}}= \\
\int d^{3} x \int \frac{d^{3} p}{(2 \pi)^{3}}[-2 \ln (2 \sin (\pi X(x)))] .
\end{array}
$$

The above relation is the principal result of this part of the paper, because contribution of Eq. (52) to the effective action $S_{e}$ completely destroys the contribution of the FaddeevPopov determinant Eq.(37) (i.e. in all orders of possible $a_{0}^{3}(x)$ expansions).

In what follows, we are going to finish the calculation of the zero-th order Schwinger term. In the first term in the right-hand side of Eq.(51) we perform first the $d^{3} p$ integration in the sense of the identity

$$
\int \frac{d^{3} p}{(2 \pi)^{3}} \frac{1}{\left(c^{2}+p^{2}\right)^{s}}=\frac{1}{\left(16 \pi^{2}\right)^{3 / 4}}\left(c^{2}\right)^{3 / 2-s} \frac{\Gamma(s-3 / 2)}{\Gamma(s)},
$$


followed by the utilization of Riemann's zeta function definition:

$$
\begin{aligned}
& \Gamma(s) \int d^{3} x \int \frac{d^{3} p}{(2 \pi)^{3}} \sum_{n=-\infty}^{+\infty} \frac{4}{\left[\left(\frac{2 \pi n}{\beta}+g A_{0}^{3}(x)\right)^{2}+p^{2}\right]^{s}}= \\
& \frac{4 \Gamma(s-3 / 2)}{8 \pi^{3 / 2}}\left(\frac{2 \pi}{\beta}\right)^{3-2 s} \int d^{3} x[\zeta(2 s-3, X)+\zeta(2 s-3,1-X)] .
\end{aligned}
$$

Applying the operation

$$
\frac{1}{2} \lim _{s \rightarrow 0} \frac{\partial}{\partial s} \frac{1}{\Gamma(s)}
$$

to the last equation and using the identity

$$
\zeta(-3, X)=-\frac{1}{4} B_{4}(X), \quad B_{4}(1-X)=B_{4}(X)
$$

where $B_{n}$ is the Bernoulli polynomial of $n$-th order, we find the final result for Eq. (54)

$$
-\frac{1}{2} \int \frac{d^{3} x}{\beta^{3}} \frac{8 \pi^{2}}{3} B_{4}(X)
$$

We can see that the first part of the final result, Eq.(52), cancels non-expanded contribution of the Faddeev-Popov determinant to the effective action. The second part of the result, Eq.(56), represents the finite contribution to the effective action.

Let us briefly discuss the "abandoned" non-interacting contributions to the effective action which have the common form

$$
S_{\text {abandon }}=\frac{1}{2} \lim _{s \rightarrow 0} \frac{\partial}{\partial s} \frac{1}{\Gamma(s)} \int_{0}^{\infty} t^{s-1} d t \int \frac{d^{3} p}{(2 \pi)^{3}} \sum_{n=-\infty}^{+\infty}\left\{\sum_{k=0}^{+\infty} \frac{(-t)^{k}}{k !} \mathcal{T}(k)\right\} .
$$

We could reexpress the above relation via combinations of hypergeometric functions. This work seems us recently laborious in the light of results expected. It is clear after some algebra, that decomposing the $A_{0}(x)$ field into the constant part and the quantum fluctuating part, so advantageous for practical calculations, the second order term in the quantum fluctuating field $a_{0}^{3}(x)$ is the lowest order term in (57). It is immediately seen, if we take into account that each term $\mathcal{T}(k)$ in Eq.(57) contains at least one commutator of the form Eq.(45), and at least one term $\mathcal{L}$ with the $A_{0}$ field. Evaluating traces of $\mathcal{T}(k)$, we find relations of the form

$$
\begin{aligned}
& \int \frac{d^{3} p}{(2 \pi)^{3}}<p|\mathcal{T}(k)| p>=\int \frac{d^{3} p}{(2 \pi)^{3}} \operatorname{tr}\{\ldots \mathcal{L} \ldots[\mathcal{M}, \mathcal{L}]\} \\
= & \ldots \int \frac{d^{3} p}{(2 \pi)^{3}} \int \frac{d^{3} q}{(2 \pi)^{3}}<p|\mathcal{L}| q>\{\mathcal{M}(q)<q|\mathcal{L}| p>-\mathcal{M}(p)<q|\mathcal{L}| p>\} .
\end{aligned}
$$

One can see that above relation differs from zero, if $A_{0}^{3}(x)$ in $\mathcal{L}$ are replaced by the quantum fluctuating field. In the other case, use of the classical constant value $A_{0}$ in $\mathcal{L}$ 
make this operator diagonal in p-representation and we find zero in Eq.(58). Therefore, the second order is the lowest order term in the quantum fluctuating field in Eq.(57) .

In the preceding discussion we have supposed that the value of the constant $A_{0}$ field is sufficiently high in comparison with Matsubara frequencies in the Fourier decomposition of space gauge potentials. Let us suppose that the value of the $A_{0}$ field is small compared to Matsubara frequencies, so that the mass of zero Fourier modes is small in comparison to masses of nonzero Fourier modes of fields. In our opinion, in this case it is not reasonable to apply Appelquist - Carazzone decoupling theorem in the same manner to all Fourier modes of space fields. Calculating the effective theory we provide the integration over nonzero Fourier modes only, leaving zero modes as dynamical variables of the effective theory. The theory defined in such a manner is a static $S U(2)$ theory in the 3-dimensional space for space gauge fields interacting with $A_{0}(x)$ field, playing the rôle of the static Higgs field. The effective potential is not periodic and possesses the global minimum for $A_{0}^{3}=0$. We find also, that the result of the cancellation of the IM and of the functional determinant resulting from the integration over nonzero Fourier modes of gauge potentials differs from the situation in the previous case.

We start from the action in Eq.(39), where we neglect the third and fourth order terms. We use the Fourier expansion of space gauge potentials as

$$
A_{i}^{a}(x, t)=\sum_{n=-\infty}^{\infty} e^{-i \frac{2 \pi n}{\beta} t} \mathcal{A}_{i}^{a}(n, x) .
$$

Integrating out nonzero Fourier modes $\mathcal{A}_{i}^{a}(n \neq 0, x)$ we obtain the nonlocal determinant which can be expanded into the sum of local terms by zeta regularization prescription and the Schwinger operator expansion.

The term of our interest is the first, non-interacting term. In what follows we use the same method of calculation as above. The important difference appears for the term corresponding to Eq.(51), which now has the form

$$
\begin{aligned}
\int_{0}^{\infty} t^{s-1} \operatorname{Tr} e^{-(\mathcal{M}+\mathcal{L}) t} d t & = \\
& \Gamma(s) \int d^{3} x \int \frac{d^{3} p}{(2 \pi)^{3}} \sum_{n=-\infty}^{+\infty}\left\{\frac{4}{\left[\left(\frac{2 \pi n}{\beta}+g A_{0}^{3}(x)\right)^{2}+p^{2}\right]^{s}}+\frac{2}{\left[\left(\frac{2 \pi n}{\beta}+g A_{0}^{3}(x)\right)^{2}\right]^{s}}\right\} .
\end{aligned}
$$

The summation $\sum^{\prime}$ means summation over $n \neq 0$. Now we can see why it is impossible to introduce the periodicity transformation (35). Introducing Riemann's zeta function, we add into each sum and subtract terms for summation index $n=0$. Executing the operation (55) we have for the second term of the last equation

$$
\int d^{3} x \int \frac{d^{3} p}{(2 \pi)^{3}}\left[-2 \ln \left(2 \sin (\pi X(x))+2 \ln \left|g A_{0}^{3}(x)\right|\right] .\right.
$$

For first term of the Eq.(59) we finally obtain

$$
-\frac{1}{2} \int \frac{d^{3} x}{\beta^{3}} \frac{8 \pi^{2}}{3}\left\{B_{4}(X)+2\left|\frac{g \beta A_{0}^{3}(x)}{2 \pi}\right|^{3}\right\} \text {. }
$$


When we compare the IM with the result (60) we find that, contrary to the periodic case, the cancellation is not complete but the new "measure" term appears

$$
M_{n}=\int d^{3} x \int \frac{d^{3} p}{(2 \pi)^{3}}\left[2 \ln \left|g A_{0}^{3}(x)\right|\right] .
$$

The partition function for the effective system is now of the form

$$
Z(\beta)=\int\left[\mathcal{D}\left(g A_{0}^{3}(x)\right)\right]\left[\mathcal{D} \mathcal{A}_{i}^{a}(n=0, x)\right] e^{-S_{e f f}+M_{n}} .
$$

The factor $\beta$ from the term $\left[\mathcal{D}\left(g A_{0}^{3}(x)\right)\right]$ disappeared due to the Fourier transform of space gauge potentials.

The present investigation gives a chance to find a reliable effective potential both for $A_{0}$ condensate and for the PL in the continuum theory. Let us sketch briefly a scheme of calculations. In the analogy with the lattice definition we introduce the following effective potential for the PL in the continuum

$$
V_{e f f}(L)=-\frac{1}{V \beta} \log \tilde{Z}(\beta)
$$

where

$$
\tilde{Z}(\beta)=\mathcal{N} \int\left[\mathcal{D} A_{\mu}^{a}(x, t)\right] \Delta_{F P}\left[f\left(A_{\mu}\right)\right] \delta\left[f\left(A_{\mu}\right)\right] \delta\left[\int \frac{d^{3} x}{\beta^{3}}(L(x)-L)\right] \exp \left[-S_{e}\right]
$$

Applying the usual method of calculations one obtains the qualitative result for the effective potential at small values of $L$

$$
V_{e f f}(L) \propto-\frac{1}{V \beta}\left[\log \operatorname{Det}^{-1 / 2} M(L)-\frac{V}{\beta^{3}} C L^{2}\right],
$$

where $C$ is a positive constant. $M(L)$ can be found in [2] where we have to use $L=\cos (X \pi), X$ corresponds to a constant part of $A_{0}(x)$. We may conclude from the last equation that despite the cancelation of the IM, the disordered contribution to the effective potential comes from the invariant distribution term. This situation is close to the lattice case studied in the previous section.

\section{Summary}

In the dimensional regularization the invariant measure does not contribute to the partition function and can be omitted from the very beginning [3, 7]. It may be not the case in other schemes of calculations. The question which we have addressed in this paper concerns the properties of the IM in the lattice regularization and in the continuum theory with zeta-functional regularization method. The main conclusions can be summarized as follows: 
1) The IM is cancelled by the integration over space gauge fields in both considered cases. In this sense the IM does not influence the confinement mechanism directly.

2) It does not follow, that the IM term can be omitted, because it cancels an important part of the gluonic kinetic energy, which tends to order the system at any temperature再.

3) Disordering contributions could appear in the partition function in the form of the invariant distribution for the expectation value of the PL.

It is emphasized that we do not expect a cancellation of the IM in QCD at zero temperature: All described effects take place in the finite temperature theory (see for discussion [10]).

The present investigation allows us to reexamine scenarios discussed in the Introduction. Our results support the scenario No.3 with nonzero $A_{0}$ condensate. Certainly, in case of absence of the IM in the definition of the partition function we would always get $A_{0}=0$, in accordance with [3]. The measure in both considered regularizations cancels a part of the gluonic energy and we have found nontrivial saddle points generating $A_{0} \neq 0$. It should be stressed that we have shown the existence of this saddle point on the lattice. It is unknown at the moment whether this saddle configuration survives the transition to the continuum limit. We expect that it is the case, because the qualitative estimate of the expectation value of the PL in the continuum has shown nontrivial minima of the periodic effective potential $L \neq \pm 1$. In the static gauge we have a simple connection between the PL and the condensate $L=\cos (X \pi)$, we hope that our expectation is real.

Let us make some further remarks on Ref.[3]. The authors of the paper have considered a partition function for the eigenvalues of the PL in the continuum theory. Their consideration is very close to ours described in the Hamiltonian formulation on the lattice as they have used the temporal gauge $A_{0}=0$ with projection onto the gauge invariant states. The conclusion of the paper is, that there is no real condensation at high temperature. The basic assumption conjectured by the authors is the cancellation of the IM term. As we showed such cancellation indeed takes place. However, in [3] the IM was not cancelled but rather simply omitted from the partition function. Presumably, it may be done in the dimensional regularization. Namely this gives a possibility to rewrite the partition function in such a form that the constant part of $A_{0}$ will be only at the imaginary unit in the exponential. After this, the conclusion $A_{0}=0$ trivially follows from the requirement of the minimum for the effective potential. The lattice and zeta-functional regularization demonstrate something different. The real cancellation of the measure makes it impossible to represent the partition function in the form proposed in [3] in these regularizations. In this case, the proof that $A_{0}=0$ obviously fails. It is clearly seen in the lattice notations. If we cancel (not omit) the IM from Eq.(17) the resulting expression cannot be rewritten as a projection operator with $\phi$ staying only at the imaginary unit in the exponential (but it is really possible if we omit the measure from (17)). Discussion of the problem whether the IM on the

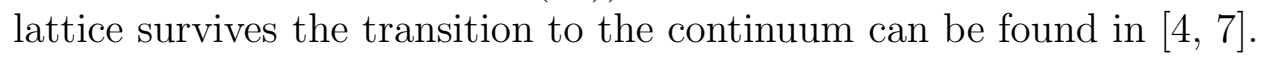

\footnotetext{
${ }^{4}$ We do not know the behaviour of this part of gluonic energy under the dimensional regularization.
} 
Our last remark concerns the result where the second order term of the IM decomposition is not cancelled and appears in the effective action [6]. In our approach, we included into the calculation the terms cubic and quartic in the gluonic fields, but only quadratic in spacelike fields of the original action. By the Gaussian integration over the spacelike fields, the timelike field appears in the functional determinant. By quantum fluctuating part decomposition of this determinant in the effective action appears the terms which cancel the corresponding invariant measure terms. In this approach we can reproduce the result of Ref. [6] if we replace the field $A_{0}^{3}(x)$ by its nonzero constant value in the determinant from the beginning of the calculation.

An idea of this work came up in Budapest during the discussions with J. Polonyi and K. Seiler. Authors are grateful to them for many fruitful remarks and explanation of their results. We wish to thank V. Petrov and G. Zinovjev for many interesting discussions and technical advises during the calculations. Also, a clear explanation of the gauge invariant nature of $A_{0}$ condensate in the continuum space-time in Nilson's identity approach provided by V. Skalozub in many private communications is appreciated. Our special gratitude is for S. Olejnik for the critical discussions and the careful reading of the manuscript.

\section{References}

[1] N. Weiss, Phys.Rev. D24 (1981) 475; Phys.Rev. D25 (1982) 2667.

[2] J. Boháčik, Phys.Rev. D42 (1990) 3554.

[3] A. Gocksch, R.D. Pisarski, Nucl.Phys. B402 (1993) 657.

[4] K. Johnson, L. Lellouch, J. Polonyi, Nucl.Phys. B367 (1991) 675.

[5] O.A. Borisenko, J Boháčik, V. Skalozub, Fortschr.Phys. 43 (1995) 301.

[6] K. Seiler, A. Schäfer, W. Greiner, Phys.Let. B350 (1995) 234; K.Sailer, W Greiner, Acta Phys. Hung.: Heavy Ion Phys. 1 (1995) 1.

[7] J. Polonyi, preprint hep-ph/9511243.

[8] J. Polonyi, K. Szlachanyi, Phys.Lett. 110B (1982) 395.

[9] O.A. Borisenko, V.K. Petrov, G.M. Zinovjev and J. Boháčik, Phase structure and confinement properties of noncompact gauge theories I, hep-lat/9508001.

[10] O.A. Borisenko, V.K. Petrov, G.M. Zinovjev, J Boháčik, Phase structure and confinement properties of noncompact gauge theories II. $Z(N)$ Wilson loop and effective noncompact model, hep-lat/9602001.

[11] V.M. Belyaev, Phys.Lett. B254 (1991) 153.

[12] J. Boháčik, P. Prešnajder, Phys.Lett. B332 (1994) 366. 
[13] A.K. Rebhan, Nucl.Phys. B430 (1994) 319.

[14] B. Svetitsky, L.G. Yaffe, Nucl. Phys. B210 (1982) 423; L.McLerran, B.Svetitsky, Phys.Rev. D24 (1981) 450; J. Kuti, J. Polonyi, K. Szlachanyi, Phys.Lett. 98B (1981) 199.

[15] M. Gross, J.F. Wheater, Nucl.Phys. B240 (1984) 253.

[16] M. Caselle, Recent Results in High Temperature Lattice Gauge Theories, heplat/9601009.

[17] C. Bernard, Phys.Rev. D9 (1974) 3312.

[18] T. Appelquist, J. Carazzone, Phys.Rev. D11 (1980) 2856.

[19] S. Hawking, Comm.Math.Phys. 55 (1977) 133.

[20] D.G.C. McKeon, T.N. Sherry, Phys.Rev. D35 (1987) 3854. 